\title{
The role of videonystagmography (VNG) in assessment of dizzy patient Soha Mekki
}

Audiology Unit, ENT Department, Faculty of Medicine, Zagazig University, Egypt

Correspondence to Soha Mekki, Professor, Audio-Vestibular Medicine, ENT Department and Head of Audiology Unit, Faculty of Medicine, Zagazig University, Zagazig University, Egypt

E-mail: mailto:Soha.mekki@hotmail.com, Soha.mekki@hotmail.com

Tel: +00201229922299; 0552362345; 0552354919

Received 17 November 2013 Accepted 25 November 2013

The Egyptian Journal of Otolaryngology 2014, 30:69-72

\begin{abstract}
Videonystagmography (VNG) is a complete diagnostic system for recording, analyzing, and reporting eye movements using video imaging technology, in which hi-tech video goggles with infrared cameras are used. VNG includes a series of tests used to determine whether a vestibular disease may be causing a balance or dizziness problem; VNG can differentiate between a central and a peripheral vestibular lesion, and, if peripheral, it can decipher between unilateral and bilateral vestibular loss. VNG addresses the functionality of each ear.
\end{abstract}

Keywords:

videonystagmography, clinical significance, subtests

Egypt J Otolaryngol 30:69-72

(c) 2014 The Egyptian Oto - Rhino - Laryngological Society $1012-5574$

\section{Introduction}

Videonystagmography (VNG) is a complete diagnostic system for recording, analyzing, and reporting eye movements using video imaging technology, in which hi-tech video goggles with infrared cameras are used.

VNG includes a series of tests used to determine whether a vestibular disease may be causing a balance or dizziness problem; VNG can differentiate between a central and a peripheral vestibular lesion, and, if peripheral, it can decipher between unilateral and bilateral vestibular loss. VNG addresses the functionality of each ear [1].

\section{Videonystagmography role in clinical decision making Videonystagmography-support diagnosis}

VNG helps document unilateral/bilateral loss of vestibular function, confirm benign peripheral positional vertigo (BBPV), and detect central lesions that are missed during a routine physical exam. VNG helps decide whether additional tests (e.g. MRI) are needed and helps in preoperative evaluation - for example, of acoustic neuroma.

\section{Videonystagmography pretest protocol}

The VNG pretest protocol includes patient interview and chart review, otoscopic ear examination, eye movement examination to modify camera configuration, and finally placement of goggles and calibration of eye movement.

\section{Videonystagmography tests}

VNG tests includes the following:

(1) Tests of oculomotor function (with fixation): includes saccade, tracking, and optokinetic tests.

(2) Tests of gaze stabilization (with or without fixation, alertness level): includes gaze/spontaneous nystagmus, static position tests.

(3) Caloric test.

(4) Tests for specific etiologies: includes Dix-Hallpike maneuver (dynamic positioning), pressure test (fistula).

(5) Others: head shake test, hyperventilation test, etc.

\section{Oculomotor tests}

\section{Saccade test}

This test assesses the ability to rapidly move the eye and refixate on the target, and appraises the latency, accuracy, and velocity of the saccade.

Abnormal saccades:

(1) Saccadic slowing: Saccadic slowing denotes the presence of a central lesion in the basal ganglia, brain stem, cerebellum, peripheral oculomotor nerves, or muscles (typically in diffuse lesions of the central pathways associated with neurodegenerative diseases); it can also be due to fatigue, drowsiness, or medication (reversible).

(2) Delayed saccades (latencies $>>200$ ms): These denote a central lesion in the frontal/ frontoparietal cortex or basal ganglia (interpreted conservatively). This condition has low clinical value if bilateral (more significant if unilateral) 
and may be caused by inattention, poor visual acuity, or medications.

(3) Saccadic dysmetria:

Hypometria: denotes a central lesion in the cerebellar flocculus.

Hypermetria: denotes a central lesion in the cerebellar vermis.

\section{Tracking (smooth pursuit)}

Tracking is the ability to smooth pursuit, which is controlled by the vestibule-cerebellum.

Abnormal tracking (pursuit):

Unilateral defective tracking (pursuit) denotes the presence of a central lesion.

A symmetric defect indicates the presence of diffuse cortical, basal ganglial, or cerebellar anomalies.

An asymmetric defect indicates the presence of focal lesions involving the ipsilateral cerebellar hemisphere, brain stem, or parieto-occipital region.

\section{Optokinetic test}

The optokinetic system is responsible for the stabilization of the visual field. VNG tests the optokinetic tracking of targets by passing a light rapidly in front of a patient from one direction to the other. Asymmetries are noted and are signs of central nervous system dysfunction.

\section{Gaze stabilization tests}

For gaze test: The patient is asked to hold his or her gaze in the right, left, up, and down positions for at least $20 \mathrm{~s}$.

If nystagmus or other abnormalities are observed in any gaze position, a re-examination at that position is conducted.

For spontaneous nystagmus test: Record the eye movements in center gaze both with and without fixation.

For static position test: Record eye movements for as long as necessary (at least $30 \mathrm{~s}$ ) to determine the presence of nystagmus. Include at least four head positions: sitting, supine, head right, and head left (for the last three positions, use the standard caloric test position with the head elevated at an angle of $30^{\circ}$ from the supine position).

Include testing in body right and body left positions if the nystagmus appears when the head is in the right/left position but is not present in the sitting and supine position to determine the effect of neck rotation.

Include any head position for which the patient has a specific complaint.

\section{Interpretation of gaze stabilization tests}

For horizontal nystagmus:

(1) Changes direction in a single gaze/head position: indicates a central lesion.

(2) Nystagmus is present with fixation and its intensity does not increase significantly (but at least doubles) without fixation: indicates a central lesion.

(3) Nystagmus is present without fixation and its intensity is less than a threshold $(4 \%)$ : normal test.

(4) All other forms of horizontal nystagmus: indicates a nonlocalizing lesion.

For vertical nystagmus:

(1) Nystagmus is present with fixation: indicates a central lesion.

(2) Nystagmus is present without fixation and its intensity is less than a threshold $\left(7^{\circ} / \mathrm{s}\right.$ in VNG): normal test.

(3) All other forms of vertical nystagmus: unknown clinical significance.

Abnormalities in the gaze stabilization tests:

Gaze-evoked nystagmus: indicates the presence of cerebellum/brain stem lesions (rule out endpoint and 'leak-through' spontaneous nystagmus).

Square wave jerk nystagmus (form of saccadic intrusion):

Abnormal only when present with fixation: indicates the presence of a central lesion in the cerebellum or basal ganglia.

Ocular flutter (form of saccadic intrusion): indicates the presence of a central lesion in the brain stem or cerebellum.

Periodic alternating nystagmus: indicates the presence of a central lesion; it is present with or without fixation and reverses direction every $2-4 \mathrm{~min}$.

Vestibular (spontaneous) nystagmus:

(1) Horizontal with or without torsional component or vertical with torsional component (in lesions involving vertical canals). 
(2) Horizontal and vertical components suppressed with fixation (intensity decreases by at least 50\%).

(3) Intensity may vary due to gaze position and alertness level (stronger when gaze is directed toward fast phases).

(4) Direction may vary in different head positions but not in different gaze positions.

(5) In the absence of fixation, the test is considered abnormal only if intensity is greater than a threshold [all forms including geotropic and ageotropic; horizontal $4 \%$ s, vertical $7 \%$ s (upbeat more common in normal individuals)].

\section{Caloric testing}

General interpretation of caloric test

(1) Bilateral weakness (BW): responses from both right and left ear less than $12 \%$ (total right ear $<12 \%$ and total left ear $<12 \%$ s).

(2) Unilateral weakness (UW\%) greater than 25\% (alternative values 20-30\%).

(3) Directional preponderance (DP\%) greater than $30 \%$ (alternative values $25-50 \%$ ).

(4) Fixation index (FI\%) greater than 60\% (alternative values $50-60 \%)$.

(5) Hyperactive: total right ear greater than $140 \%$ or total left ear greater than $140^{\circ} / \mathrm{s}$.

\section{Abnormal calorics}

Unilateral weakness (canal paresis): It denotes a peripheral vestibular lesion involving the lateral (horizontal) canal or its afferent pathways on the side of the weaker response (involved pathway extends from the end organ to the root entry zone of the vestibular nerve in the brain stem).

In the acute phase, significant spontaneous nystagmus is present.

It can be caused by diseases that affect the labyrinth, the vestibular nerve, or the blood supply to these sites.

Central lesions that affect the root entry zone of the vestibular nerve (e.g. multiple sclerosis [MS]) can cause unilateral weakness but other central nervous system signs may also be present.

Bilateral weakness: When bilateral caloric weakness is present, an additional test (rotation chair, active head rotation, head thrust, or bilateral ice water tests) is needed to determine whether true bilateral vestibular lesion or hyporesponsiveness exists. Hyporesponsiveness (BW) denotes either peripheral vestibular lesion in both ears or a central lesion.
Caloric-induced vertical nystagmus: This condition is present in normal individuals as well as in patients with various disorders most likely due to stimulation of posterior/anterior canals.

Caloric perversion: All four irrigations must generate purely vertical nystagmus or vertical nystagmus must be much stronger than horizontal nystagmus, which is a central finding but extremely rare.

\section{Dix-Hallpike}

Dynamic positioning nystagmus may be an indication of a positive test. It entails a transient nystagmus that is provoked by the movement of the head to a critical position.

\section{Abnormal Dix-Hallpike BBPV nystagmus type}

Torsional-vertical nystagmus (usually upbeat and torsion toward the undermost ear indicating posterior canal BPPV): usually delayed onset, transient, fatigable with subjective sensation of vertigo.

General interpretation of dynamic position test:

A static positional nystagmus is present as long as the head remains in the critical position.

What if nystagmus has no torsional component? If purely vertical nystagmus, then not BPPV. If purely horizontal nystagmus, then perform roll maneuver for horizontal canal BBPV.

Diagnosis of BPPV:

Posterior/anterior semicircular canal BPPV

\begin{tabular}{lll}
\hline & \multicolumn{1}{c}{ Right torsion } & \multicolumn{1}{c}{ Left torsion } \\
\hline Up beat & Right posterior canal & Left posterior canal \\
Down beat & Right anterior canal & Left anterior canal \\
\hline
\end{tabular}

Horizontal semicircular canal BPPV diagnosed by roll test

\begin{tabular}{|c|c|c|}
\hline & Head right & Head left \\
\hline $\begin{array}{l}\text { Canalithiasis } \\
\text { (geotropic) }\end{array}$ & $\begin{array}{l}\text { Pure horizontal right } \\
\text { beat }\end{array}$ & Pure horizontal left beat \\
\hline $\begin{array}{l}\text { Cupulolithiasis } \\
\text { (ageotropic) }\end{array}$ & Pure horizontal left beat & $\begin{array}{l}\text { Pure horizontal right } \\
\text { beat }\end{array}$ \\
\hline
\end{tabular}

\section{Other tests}

\section{Head-shaking test}

The head-shaking test is used for the detection of latent spontaneous vestibular nystagmus. The patient's eyes are observed for nystagmus immediately after a passive rapid head shaking along a vertical axis. In peripheral vestibular disturbances, the induced nystagmus can be classified into 
deficiency-type nystagmus, recovery-type nystagmus, and biphasic nystagmus, which is usually a mixture of the two.

\section{Acknowledgements \\ Conflicts of interest}

None declared.

\section{References}

1 Barber HO, Stockwell CW. Manual of electronystagmography (1st ed.). St. Louis, MO: Mosby 1976.
2 Bhattacharyya N, Baugh RF, Orvidas L, Barrs D, Bronston LJ, Cass S, Haidari J. Clinical practice guideline: Benign paroxysmal positional vertigo. Otolaryngology - Head \& Neck Surgery, 2008; 139 (5 Suppl. 4):S47-S81.

3 Furman JM, Cass SP. Benign paroxysmal positional vertigo. New England Journal of Medicine, 1999; 341:1590-1596.

4 Campbell K. Essential Audiology for Physicians. San Diego: Singular Publishing Group; 1997.

5 Jacobson GP, Newman CW, Kartush JM. Handbook of Balance Function Testing. San Diego: Singular Publishing Group; 1993.

6 McCaslin DL, Jacobson GP. Current Role of The Videonystagmography Examination in the Context of the Multidimensional Balance Function Test Battery. Seminars in Hearing 2009; 30:242-253.

7 Shepard NT, Telian SA. Practical Management of the Balance Disorder Patient. San Diego: Singular Publishing Group; 1996. 